\title{
Prevalence of consanguinity among ASD individuals: Systematic Review and Meta-analysis
}

\section{Introduction}

Autism Spectrum Disorder (ASD) is a complex neurodevelopmental disorder that affects the person's capability to socially interact with others, communicate and reply to stimulations in their environments. It is having been estimated that out of every 1000 child, $3-6$ of them would have autism worldwide and the prevalence is higher in males compared to females. Studies have found that autism prevalence is $0.6 \%$ in the Kingdom of Saudi Arabia, $1.4 \%$ in Oman and $1.14 \%$ in Qatar. Although neonatal and prenatal risk factors were the focus of numerous epidemiological studies over 40 years, Autism etiology is still unknown. One of the factors that have been linked to autism and is related to genetic is consanguinity. Almost 20\% of the world's population lives in societies that favor consanguineous. In Qatar, consanguinity is estimated to be $54 \%$.

With the growing worldwide prevalence of ASD, as well as in Qatar (1.14\%), research should have rapidly progressed efforts to improved understand the increase in it is incidence and co morbidities.

\section{Aim}

We aim to estimate the global prevalence of consanguinity among the ASD families and compare it that among different populations.

\section{Methods and Materials}

\section{Meta-Analysis of observational studies} reporting prevalence of consanguinity among ASD families were searched systematically in important databases including EMBASE, PubMed and Academic Search Complete. Individual studies were screened by two reviewers independently, extracted data and assessed the risk of bias using a risk of bias tool (Hoy's tool). Random Effect model was used to calculate pooled weighted estimates due to considerable heterogeneity. Subgroups analysis was also calculated.
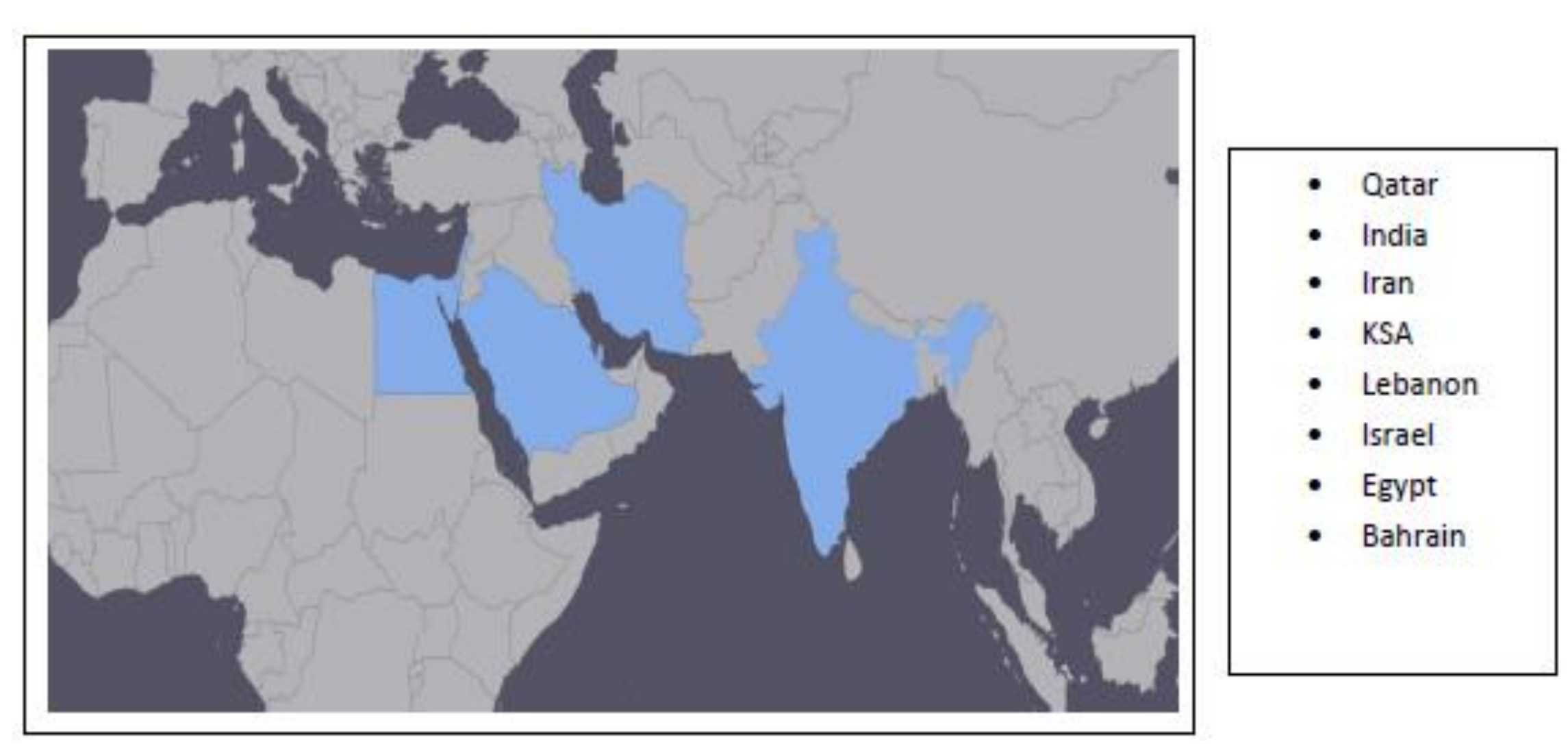

Figure 1. Geographic location of included studies .

\section{Results}

10 publications were identified based on our inclusion criteria from 8 different countries, 4 of them were from the Gulf Cooperation Council (GCC) and the rest were from: Lebanon (2 studies), Egypt, Jordan, Iran, and Israel). Studies varied in ASD cases numbers as it ranged between $49-500$, and the total ASD individuals in all studies were 1581. All studies address consanguinity among the ASD families despite the variation in the methods.

The pooled estimate of consanguinity among ASD families was $24 \%$. Subgroup analysis by the study country led to a higher pooled estimate of consanguinity of $38 \%$ in the GCC countries compared to other than GCC countries with a pooled estimate of $16 \%$. Also, the overall odd ratio calculated from the case-control studies included in our meta-analysis was 1.5 .

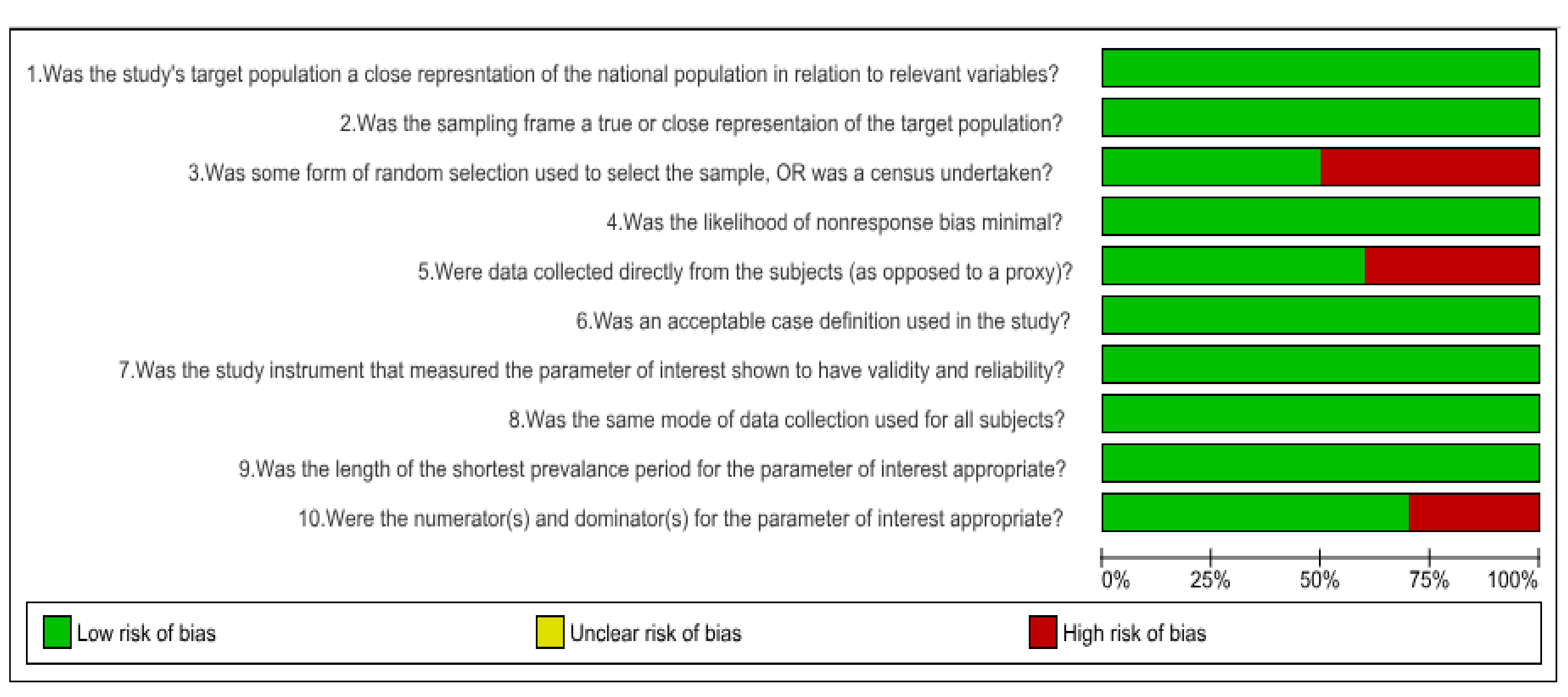

Figure 2. Risk of Bias graph

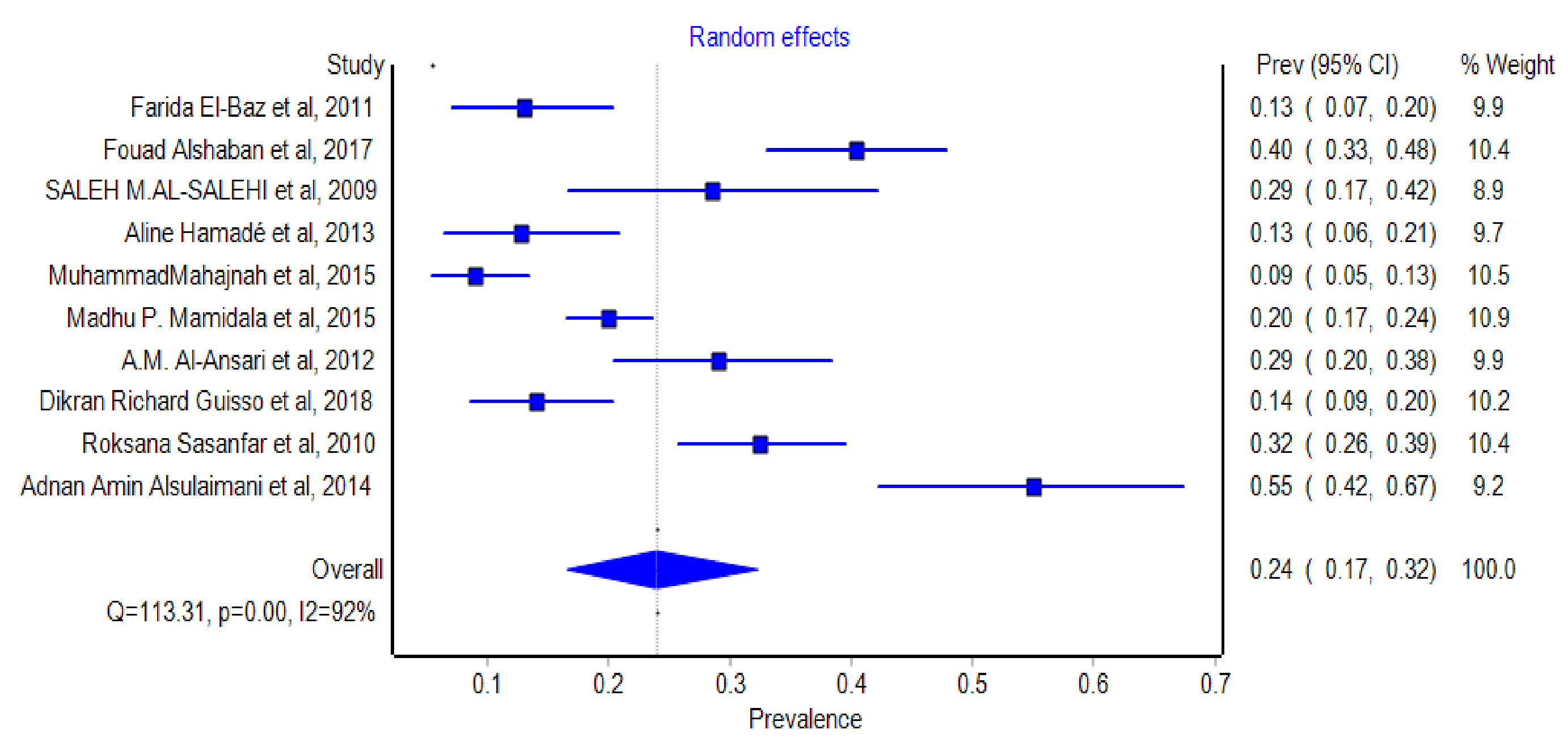

Figure 3. The overall pooled prevalence of consanguinity among ASD patients.

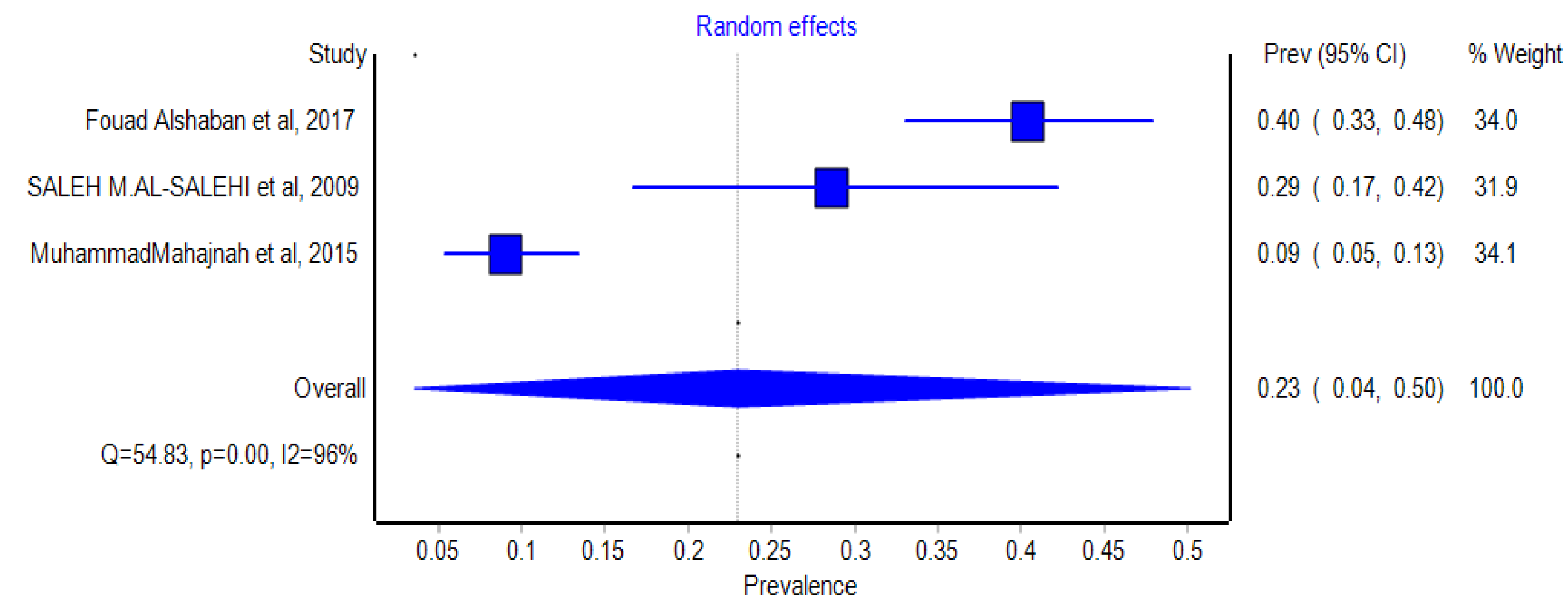

Figure 4. The pooled prevalence of consanguinity among ASD patients in cross-sectional studies

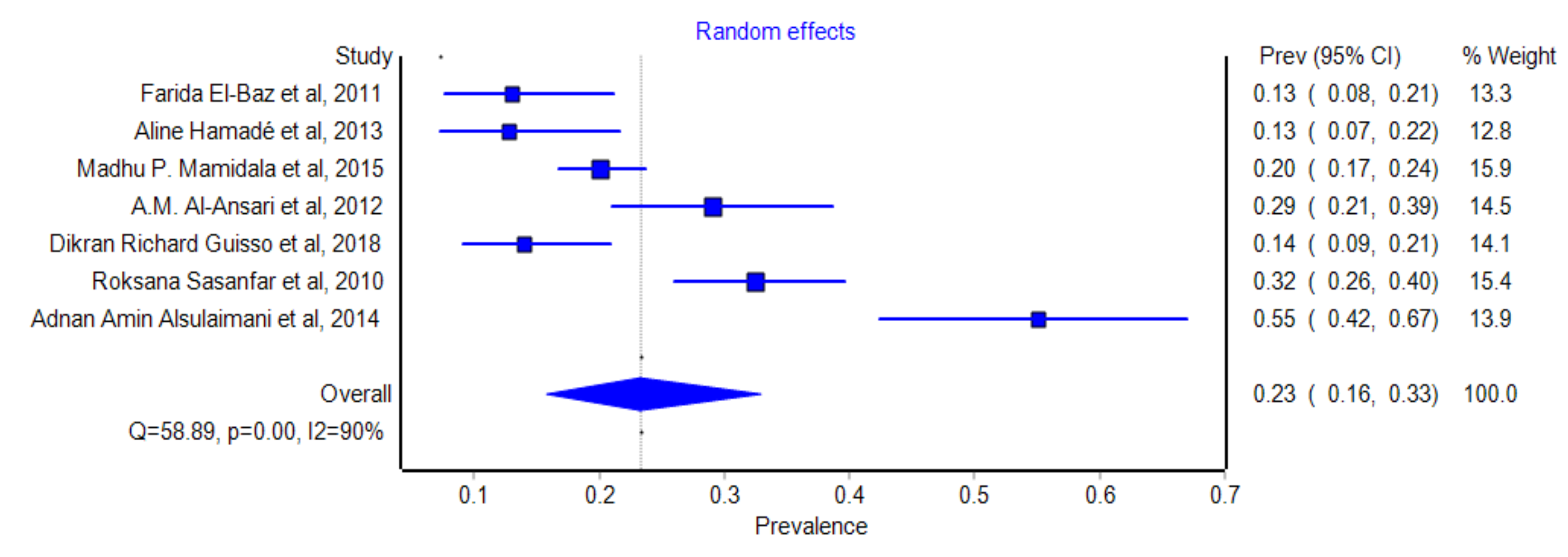

Figure 5. The pooled prevalence of consanguinity among ASD patients in the case-control studies

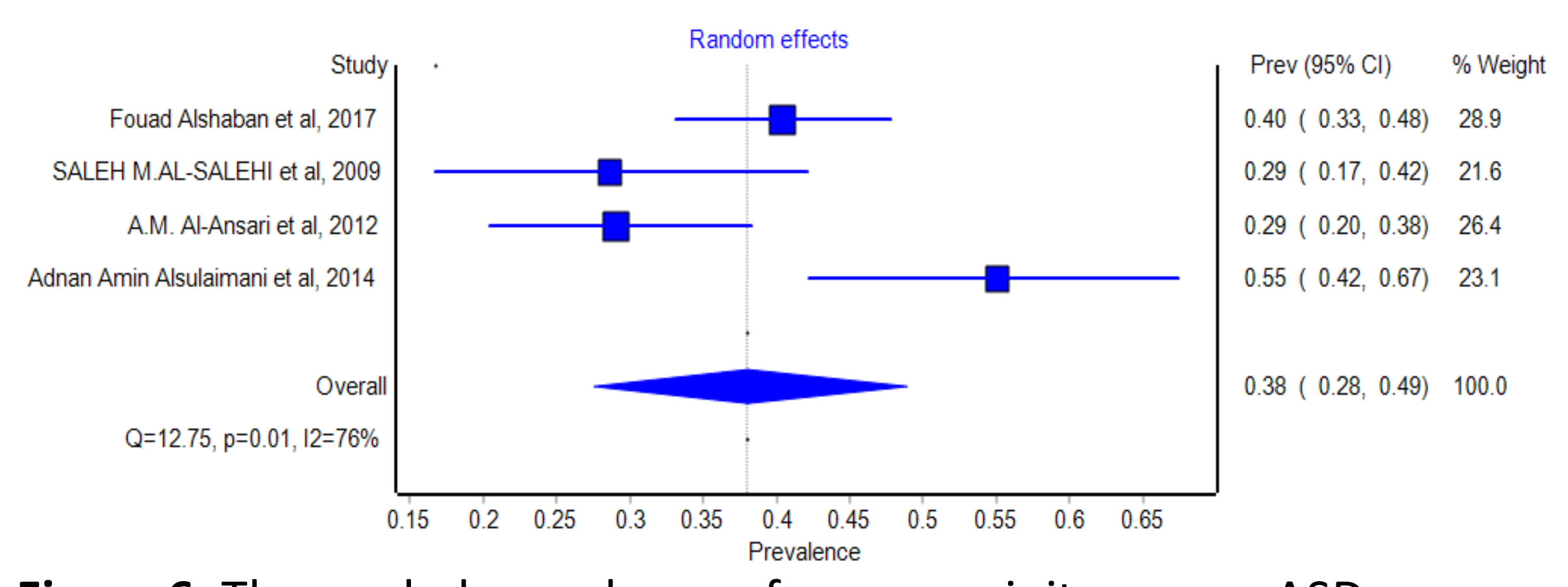

Figure 6. The pooled prevalence of consanguinity among ASD patients in the GCC countries.

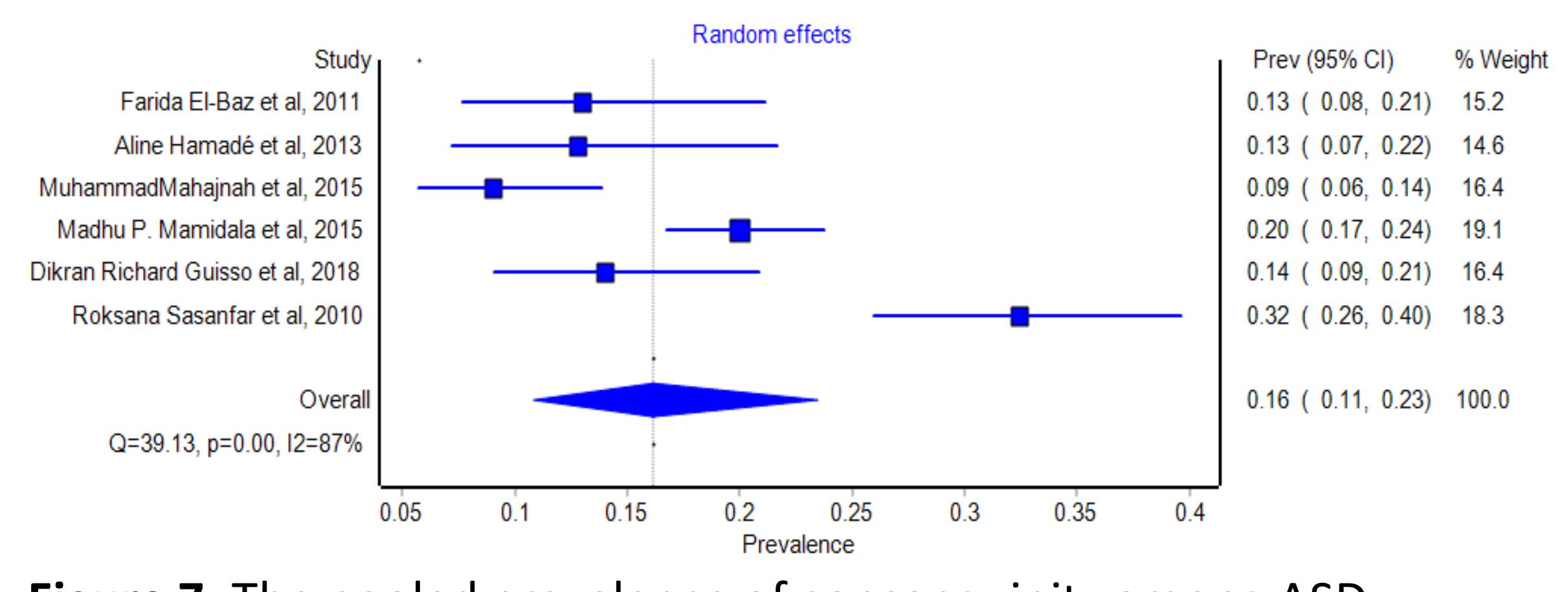

Figure 7. The pooled prevalence of consanguinity among ASD patients in the other than GCC countries

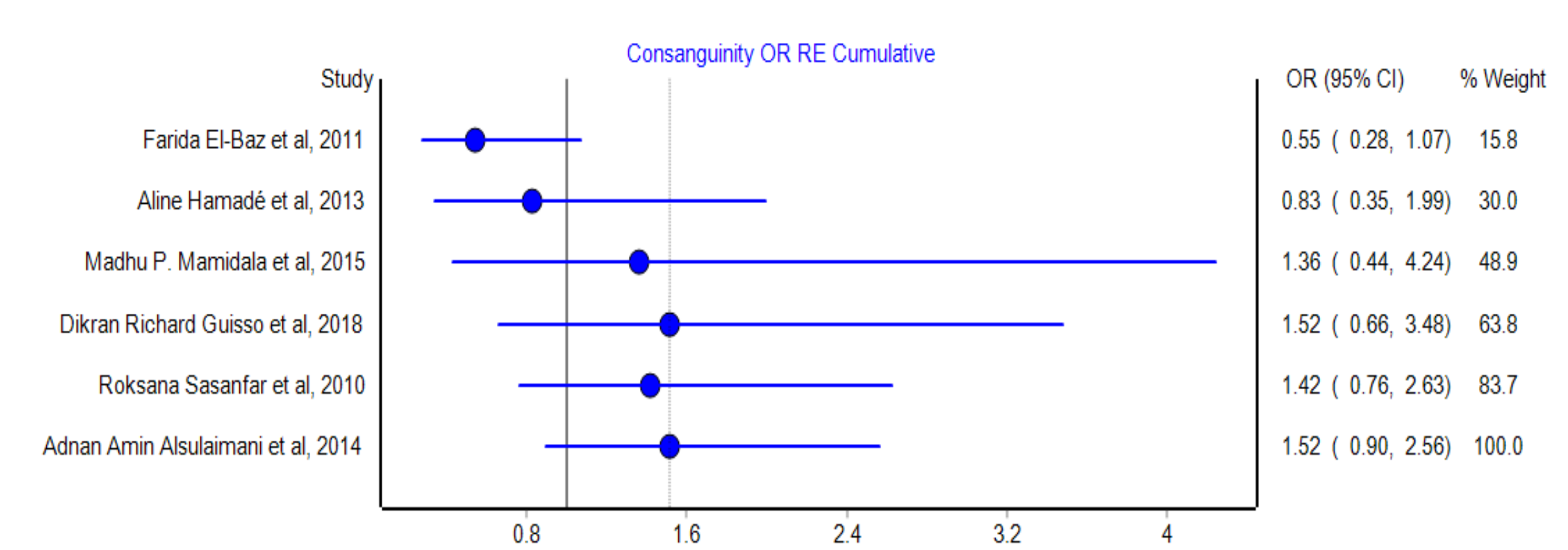

Figure 8. The overall pooled odd ratio of consanguinity among ASD patients and controls.

\section{Discussion}

To our knowledge, this is the first meta-analysis that studied the prevalence of consanguinity among ASD families worldwide. Children born to consanguineous parents have been reported to have lower social behavior and cognitive ability, which are the main problems with ASD children. Our study qualitatively reviewed the prevalence of consanguinity among ASD families throughout the world and 10 eligible studies from eight countries were identified. We quantitatively synthesized the results and the key findings of this study showed a $24 \%$ overall pooled estimate of consanguinity among ASD families. GCC countries showed a high estimated pooled prevalence of consanguinity among ASD families as $38 \%$ compared to countries other than GCC $16 \%$, which was higher than our overall pooled prevalence. This high estimate among GCC countries can be related to the high rates of consanguineous marriage in the GCC countries compared to the worldwide rates $(20 \%)$. In Qatar the rate of consanguineous marriage reported as 54\%. This high rates among GCC countries may be due to factors like rooted cultural beliefs, social life and customs in addition to, economic benefits of keeping wealth within the families.

\section{Conclusions}

The globally estimated pooled consanguinity prevalence among ASD patients was $24 \%$, GCC countries showed a higher pooled prevalence $(38 \%)$

\section{Contact}

Dr. Saba Elhag Qatar University

Email: sabaelmubarak@gmail.com

\section{References}

(2012). Global Prevalence of Autism and Other Pervasive Developmental Disorders. Autism Research, 5(3), 160-179. doi:10.1002/aur.239

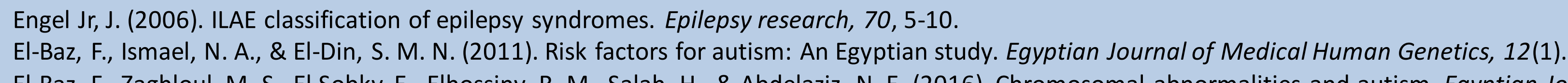
Human Genetics, 17(1), 57-62 Alshaban, F., Aldosari, M., El Sayed, Z., Tolefat, M., El Hag, S., Al Shammari, H.,.... Fombonne, E. (2017). Autism spectrum disorder in Qatar: Profiles and correlates of a large clinical sample. Autism \& Developmental Language Impairments, 2, 2396941517699215.
Al-Farsi, Y. M., Al-Sharbati, M. M., Al-Farsi, O. A., Al-Shafaee, M. S., Brooks, D. R., \& Waly, M. I. (2011). Brief report: Prevalence of autistic spectrum disorders

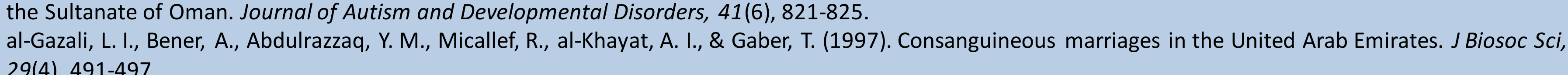

